\title{
Ganho de peso de codornas europeias mantidas em ambiente controlado
}

\author{
Productive performance of european quails kept in controlled environment \\ Ganancia de peso de la codorniza europea mantenida en un entorno controlado
}

Recebido: 23/08/2021 | Revisado: 03/09/2021 | Aceito: 07/09/2021 | Publicado: 09/09/2021

\author{
Márcia Ederlinda Souza Barbosa \\ ORCID: https://orcid.org/0000-0002-5354-009X \\ Universidade Federal de Campina Grande, Brasil \\ E-mail: m-ederlinda@hotmail.com \\ Diego José Araújo Bandeira \\ ORCID: https://orcid.org/0000-0002-6245-9875 \\ Universidade Federal de Campina Grande, Brasil \\ E-mail: diegoimperium8@gmail.com \\ Vitória Ediclecia Borges \\ ORCID: https://orcid.org/0000-0001-6279-350X \\ Universidade Federal de Campina Grande, Brasil \\ E-mail: kecinha_tgs@hotmail.com \\ Ariadne Soares Meira \\ ORCID: https://orcid.org/0000-0001-8740-9009 \\ Universidade Federal de Campina Grande, Brasil \\ E-mail: ariadnesm_eng@ @otmail.com \\ Thiago Galvão Sobrinho \\ ORCID: https://orcid.org/0000-0003-0871-9119 \\ Universidade Federal de Campina Grande, Brasil \\ E-mail: galvaosobrinho@gmail.com \\ José Pinheiro Lopes Neto \\ ORCID: https://orcid.org/0000-0003-4960-5679 \\ Universidade Federal de Campina Grande, Brasil \\ E-mail: lopesneto@gmail.com
}

\begin{abstract}
Resumo
O conforto térmico é essencial para a produção animal influenciando diretamente a conversão alimentar e aumento na produção. O experimento foi realizado no Laboratório de Construções Rurais e Ambiência - LACRA, da Universidade Federal de Campina Grande. Sendo conduzido em duas fases com duração de 32 dias cada. Foram utilizadas 768 codornas europeias (Cortunix cortunix cortunix). Utilizou-se 2 baterias de gaiolas, contendo em cada 6 gaiolas e, estas divididas em dois boxes, totalizando 12 boxes por bateria. Foram simuladas temperaturas ambientais $\left(25{ }^{\circ} \mathrm{C}\right.$ e $\left.29{ }^{\circ} \mathrm{C}\right)$ para densidade populacional de $\left(70\right.$ aves $/ \mathrm{m}^{2}$ e 90 aves $\left./ \mathrm{m}^{2}\right)$ nas duas faixas de temperatura, sendo a umidade relativa igual para ambas às etapas, em torno dos $60 \%$. Foi analisado o desempenho no ganho de peso sob tais circunstâncias, onde a diferença na densidade populacional não apresentou interferência significativa, entretanto, ocorreu uma diferença de $20 \mathrm{~g}$ no ganho de peso médio por animal quando na temperatura inferior.
\end{abstract}

Palavras-chave: Codornas; Desempenho; Temperatura.

\begin{abstract}
Thermal comfort is essential to animal production directly influencing feed conversion and increase in production. The experiment was conducted at the Laboratory of Rural Buildings and Ambience-seals, the Federal University of Campina Grande. Is conducted in two stages lasting 32 days each. 768 European quail (Cortunix cortunix cortunix) were used. We used 2 battery cagels, each containing 6 cages and they split into two boxes, totaling 12 boxes per battery. Environmental temperatures $\left(25^{\circ} \mathrm{C}\right.$ and $\left.29^{\circ} \mathrm{C}\right)$ to population density $\left(70\right.$ birds $/ \mathrm{m}^{2}$ and 90 birds $\left./ \mathrm{m}^{2}\right)$ in the two temperature ranges were simulated, and the relative humidity equal for both the stages, around $60 \%$. Performance in weight gain under such circumstances, where the difference in population density no significant interference occurred, however, a difference of $20 \mathrm{~g}$ in average weight gain per animal was analyzed as the lower temperature.
\end{abstract}

Keywords: Quail; Performance; Temperature.

\section{Resumen}

El confort térmico es esencial para la producción animal, ya que influye directamente en la conversión alimenticia y el aumento de la producción. El experimento se llevó a cabo en el Laboratorio de Ambientes y Construcciones Rurales LACRA, de la Universidad Federal de Campina Grande. Se realiza en dos fases de 32 días cada una. Se utilizaron 768 codornices europeas (Cortunix cortunix cortunix). Se utilizaron dos jaulas en batería, cada una con 6 jaulas y estas se dividieron en dos cajas, totalizando 12 cajas por batería. Se simularon temperaturas ambientales $\left(25^{\circ} \mathrm{C}\right.$ y $\left.29^{\circ} \mathrm{C}\right)$ para 
densidad poblacional (70 aves $/ \mathrm{m}^{2}$ y 90 aves $/ \mathrm{m}^{2}$ ) en ambos rangos de temperatura, siendo la humedad relativa igual para ambas etapas, alrededor del 60\%. Se analizó el comportamiento en la ganancia de peso en tales circunstancias, donde la diferencia en la densidad de población no presentó interferencia significativa, sin embargo, hubo una diferencia de $20 \mathrm{~g}$ en la ganancia de peso promedio por animal cuando a menor temperatura.

Palabras clave: Codorniz; Rendimento; Temperatura.

\section{Introdução}

A falta de bem estar e conforto térmico interfere no mecanismo termodinâmico que as aves possuem para se protegerem de extremos climáticos, acarretando no desperdício de energia e consequentemente na redução do consumo, menor taxa de crescimento, alteração na conversão alimentar e queda na produção (Abreu \& Abreu, 2003; Jordão Filho, 2011; Guimarães, 2012).

Existem fatores essenciais para uma boa criação de codornas, onde o produtor é responsável pelo desenvolvimento da cadeia produtiva, desde a higienização do local, água servida de boa qualidade e fresca, alimentação de acordo com a idade, instalações que proporcione um conforto e manejo apropriado às aves (Bertechini, 2010; Dias, 2012). A questão do bem-estar dos animais e a utilização dos recursos naturais, segundo Silva e Miranda (2009) serão relevantes no contexto da produção mundial de alimentos.

As codornas são resistentes e se adaptam a diversas condições ambientais, mas possuem um melhor desenvolvimento em clima estável e, locais protegidos contra intempéries (Rodrigues, 2006; Lanna, 2012). Pesquisas direcionadas a avicultura tem evoluído consideravelmente nos últimos tempos, em todos os seus aspectos, transformando a atividade de produção de carne e ovos em uma desenvolvida indústria de alimentos (UBA, 2001; Souza, 2013).

O consumo da carne de codorna é muito difundido em algumas regiões por ser uma fonte alternativa de proteína com excelência de qualidade, em função do seu sabor, o que levou a sua produção a ser, segundo Barreto (2007), uma opção promissora para o setor avícola.

A coturnicultura passou a ser um negócio promissor para o futuro, após o investimento pelos matrizeiros em seleção e qualidade das aves. Foram surgindo daí alojamentos maiores, em galpões, com tecnologia mais avançada, com resultados que deram lugar ao crescimento, (Gomes, 2010; Melo, 2013).

No Brasil são duas as linhas de codorna criadas, sendo a coturnix coturnix japonica voltada exclusivamente para a produção de ovos e a coturnix coturnix coturnix, esta de origem europeia, voltada tanto para a produção de carne quanto de ovos, entretanto, a exploração de codornas tipo carne vem se constituindo em nova alternativa para o setor avícola (Corrêa et al., 2005; Oliveira, 2011; Monte et al. 2018). São vários os centros de pesquisa públicos que se dedicam a trabalhos de melhoramento genético visando uma melhor eficiência produtiva das codornas (Murakami \& Garcia, 2006).

A criação de codornas é uma atividade economicamente crescente e com destaque, segundo Silva et al. (2011), o Brasil ocupa o quinto lugar na produção de carne de codorna no cenário mundial. Na Paraíba, ocupa o $4^{\circ}$ lugar (7,43\%) no ano de 2008 em produção de ovos e, em 2009 passou a ocupar o $3^{\circ}$ lugar (9,18\%), com um incremento de 1,75\% (IBGE, 2009). Com isso há necessidade de ampliar estudos na criação de codornas de corte, portanto, objetivou-se avaliar o desempenho produtivo de codornas europeias mantidas em ambiente controlado.

\section{Metodologia}

\section{Localização do Experimento}

O experimento foi realizado no Laboratório de Construções Rurais e Ambiência- LACRA, da Universidade Federal de Campina Grande. Sendo conduzido em duas fases com duração de 32 dias cada em câmara climática. A câmara climática é composta por iluminação interior de luz fluorescente comum por meio de 4 lâmpadas de 5 watts, um exaustor e um 
climatizador. Foram utilizadas 768 codornas europeias (cortunix cortunix cortunix), com 10 dias de idade sem sexagem prévia, onde as mesmas ficaram uma semana em fase adaptativa no piso da câmara climática com cobertura de casca de arroz. Após adaptação foram relocadas em gaiolas galvanizadas forrada com jornal no piso, foram alocadas bandejas com jornal e maravalha abaixo do piso para recebimento dos excrementos (fezes e urinas) das aves, sendo trocadas a cada dois (02) dias. Utilizou-se 2 baterias de gaiolas, contendo em cada 6 gaiolas e, estas divididas em dois boxes, totalizando 12 boxes por bateria. As dimensões das gaiolas são de 1,00 m x 0,40 m x 0,25 m (comprimento, largura e altura), respectivamente. Foram simuladas temperaturas ambientais $\left(25{ }^{\circ} \mathrm{C}\right.$ e $\left.29{ }^{\circ} \mathrm{C}\right)$ para densidade populacional de $\left(70\right.$ aves $/ \mathrm{m}^{2}$ e 90 aves $\left./ \mathrm{m}^{2}\right)$ nas duas faixas de temperatura, sendo a umidade relativa igual para ambas às etapas, em torno dos $60 \%$. A ração utilizada de formulação comercial, tanto na fase inicial quanto na de engorda, a água ofertada foi da rede de abastecimento público, ambos servidos à vontade. A cada segunda feira, pesava 10 aves por gaiola que foram etiquetadas anteriormente e, pesava o restante do lote para avaliação final das densidades. Pesou-se semanalmente para avaliação do desempenho produtivo como forma de avaliar o ganho máximo de peso de abate.

\section{Disposição das baterias de gaiolas}

As baterias foram identificadas na parte frontal em Tratamentos, dispostas em tratamento 1 ( $\mathrm{T}$ 1) e tratamento 2 ( $\mathrm{T}$ 2). As gaiolas foram enumeradas, iniciando de cima para baixo, com etiquetas plastificadas e presas com cordão de sisal de acordo com a densidade e repetição em cada densidade. Dessa forma, a bateria com a densidade D1 foi identificada de cima para baixo da seguinte forma: D1 R1, D1 R2, D1 R3, D1 R4, D1 R5, D1 R6. A segunda bateria teve a seguinte identificação D2: D2 R1, D2 R2, D2 R3, D2 R4, D2 R5 e D2 R6. Estas identificações permaneceram durante as duas temperaturas estudadas. Os identificadores R1, R2, R3, R4, R5 e R6 correspondem às repetições para cada densidade de criação escolhida.

As baterias foram colocadas lado a lado, sendo que a bateria com D1 disposta próxima a porta de entrada da câmara climática, a outra ficando no final da câmara (Figura 1).

Figura 1(A). Bateria com seis gaiolas. 1(B) Detalhamento das gaiolas.
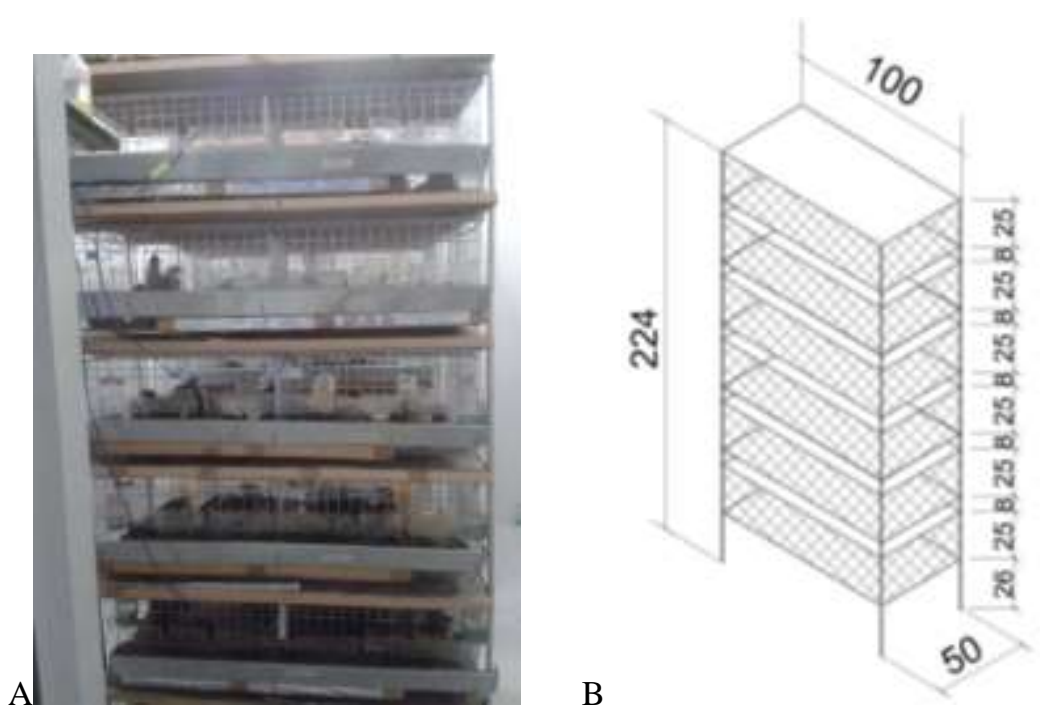

Fonte: Acervo dos autores. 


\section{Câmara climática}

O experimento foi conduzido no interior de uma câmara climática, onde a mesma possui controle eletrônico de temperatura e umidade do ar. Possui área de $5,7 \mathrm{~m}^{2}$ e pé direito de $2,65 \mathrm{~m}$, sendo confeccionada em chapas de aço laminado, com proteção anti corrosiva e preenchimento em isopor de alta densidade, com $10 \mathrm{~cm}$ de espessura (Figura 2).

Figura 2(A). Layout interno da câmara climática 2(B). Vista externa.

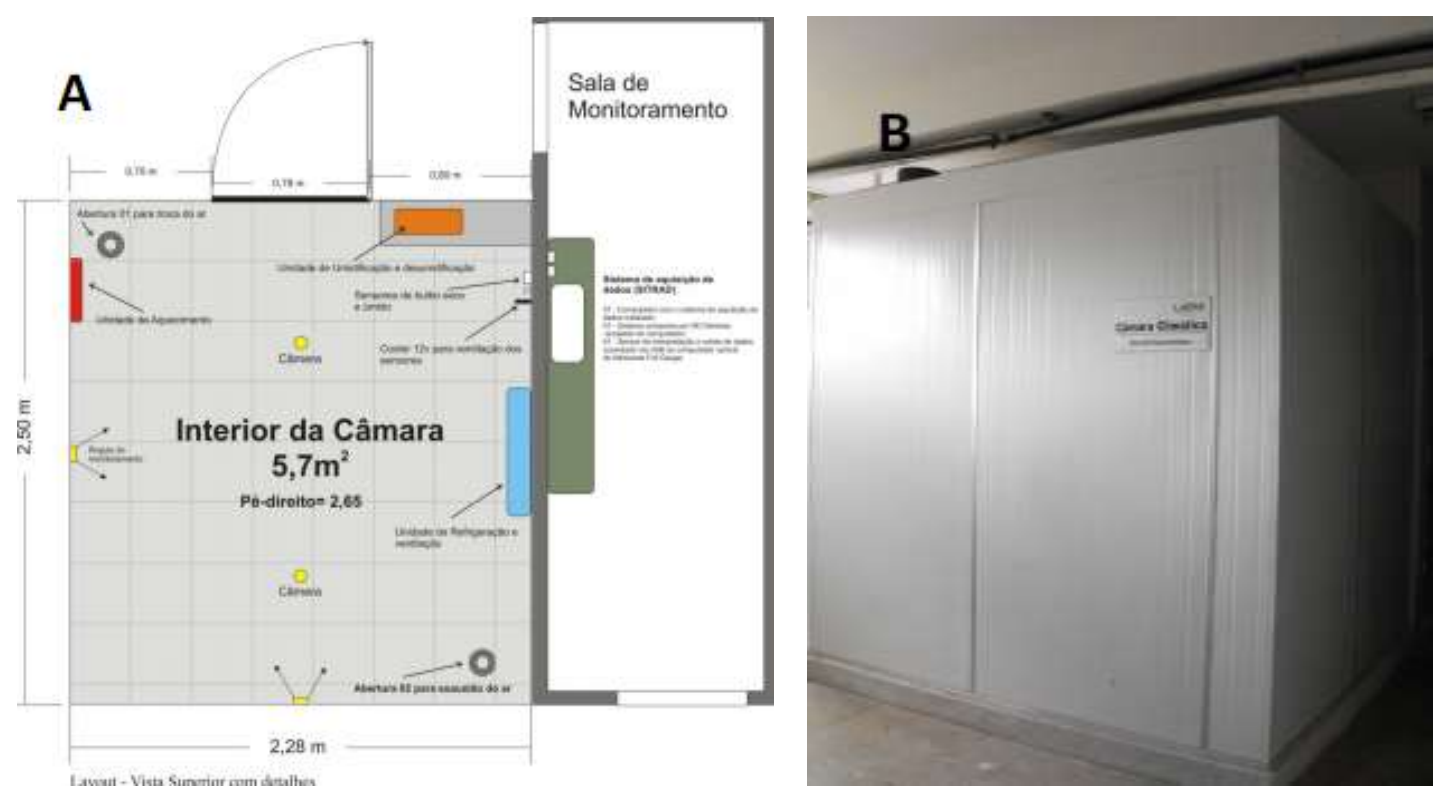

Fonte: Acervo dos autores.

No interior da câmara havia um sistema integrado de equipamentos, sendo uma unidade de refrigeração (Figura 3A) da marca Fujitsu -Inverter - de 18.000 btu/h (SPLIT), uma unidade de umidificação e desumidificação (Figura 3B), um exaustor localizado no teto da câmara para realizar a renovação do ar (Figura 3C), uma unidade de aquecimento (Figura 3D) da marca Cadence e 04 (quatro) lâmpadas fluorescentes comuns de cinco watts, acopladas em spots tipo tartarugas, ficando acesas por todo o período (dia e noite), como estímulo alimentar e hídrico. 
Figura 3. Equipamentos de controle no interior da câmara climática
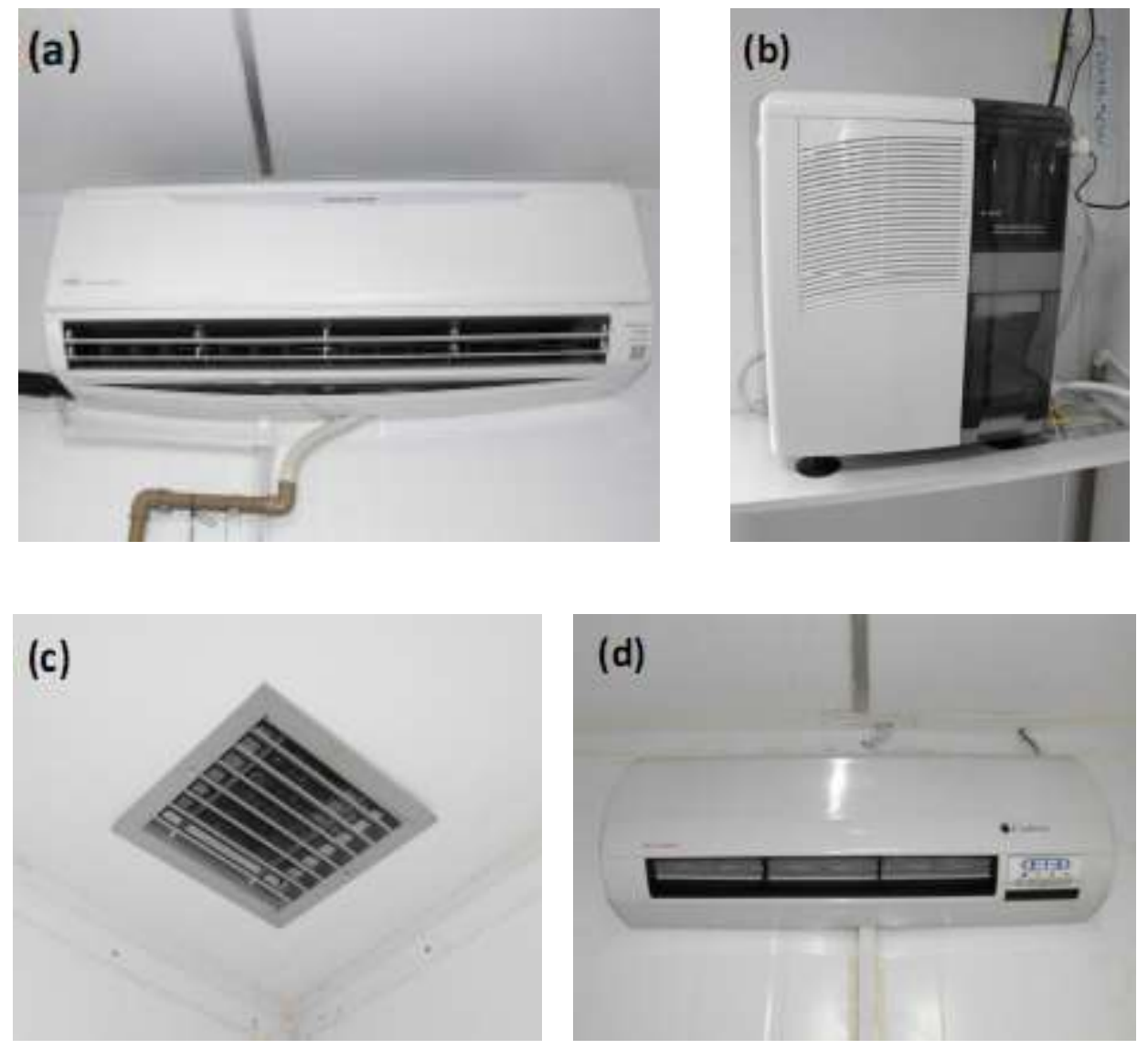

Fonte: Acervo dos autores.

\section{Ração}

A ração foi adquirida juntamente com as codornas, na Granja Natal, no município de Puxinanã - PB. A composição das rações foi elaborada no mesmo local, sendo apresentada no Quadro 1 a composição da ração inicial. Nos Quadros 2, 3 e 4 são apresentados o Premix da ração inicial, a composição da ração de engorda e o Premix da ração de engorda, respectivamente, no quadro 5 o relatório de análises bromatológicas, realizado pelo laboratório de análise de alimentos do departamento de zootecnia da Universidade Federal da Paraíba

Quadro 1. Composição da ração inicial.

\begin{tabular}{|cc|}
\hline INSUMOS & QUANTIDADE Kg \\
\hline Milho & 153,4 \\
Soja & 120 \\
Óleo & 6,6 \\
Sal & 1 \\
Premix & 20 \\
\hline TOTAL & 301 \\
\hline
\end{tabular}


Research, Society and Development, v. 10, n. 11, e540101119753, 2021

(CC BY 4.0) | ISSN 2525-3409 | DOI: http://dx.doi.org/10.33448/rsd-v10i11.19753

Quadro 2. Composição do Premix da ração inicial.

\begin{tabular}{|cc|}
\hline INSUMOS & QUANTIDADE Kg \\
\hline Macrovita & 24 \\
Milho & 14,9 \\
Max Bind & 0,8 \\
Colistina & 0,12 \\
Bacitracina & 0,1 \\
Coccitec & 0,08 \\
\hline TOTAL & 40 \\
\hline
\end{tabular}

Fonte: Granja Natal (2014).

Quadro 3. Composição da ração de engorda.

\begin{tabular}{|cc|}
\hline INSUMOS & QUANTIDADE Kg \\
\hline Milho & 188,6 \\
Soja & 93,7 \\
Farinha de carne & 10,7 \\
Óleo & 2 \\
Premix & 4 \\
Calcário (fino) & 3 \\
\hline TOTAL & 302 \\
\hline
\end{tabular}

Fonte: Granja Natal (2014).

Quadro 4. Composição do Premix da ração de engorda.

\begin{tabular}{|cc|}
\hline INSUMOS & QUANTIDADE Kg \\
\hline Sal comum & 5 \\
Natuphos & 0,15 \\
Cenzayme & 1,5 \\
Metionina & 0,6 \\
Lisina & 2,5 \\
Max Bind & 3 \\
Colistina & 0,6 \\
Bacitracina & 1,5 \\
Milho & 10,15 \\
\hline TOTAL & 40 \\
\hline
\end{tabular}

Fonte: Granja Natal (2014). 
Quadro 5. Relatório de análises bromatológicas.

\begin{tabular}{|ccccc|}
\hline Amostras & $\mathrm{MS}^{1}$ & $\mathrm{MM}^{2}$ & $\mathrm{~PB}^{3}$ & $\mathrm{EE}^{4}$ \\
\hline Ração Inicial & 88,89 & 5,63 & 26,87 & 4,17 \\
Ração Engorda & 88,37 & 5,32 & 23,06 & 7,19 \\
\hline & 1 -Matéria Seca. 2 - Matérias Mineral. 3 - Proteína Bruta. 4 - Extrato Etéreo & \\
\hline
\end{tabular}

Fonte: Laboratório de análise de alimentos do departamento de zootecnia da Universidade Federal da Paraíba.

\section{Resultados e Discussão}

A temperatura ambiente influenciou no ganho de peso, ocasionando uma diferença mínima em relação as aves mantidas em temperatura termoneutra. Quanto à diminuição no ganho de peso ocorrida na T2 $\left(30,8^{\circ} \mathrm{C}\right)$, em parte, pode ser explicada pela redução no consumo de ração e aumento da ingestão de água. Sendo que não ocorreu efeito significativo na conversão alimentar nas duas fases avaliadas, podendo observar na Tabela 1 os dados obtidos pela análise da ANOVA, quanto ao ganho de peso.

Tabela 1. Quadro de análise de variância do Ganho de Peso (GP) em função das diferentes temperaturas (F1), densidades de alojamento (F2) e alturas de gaiolas estudadas (F3).

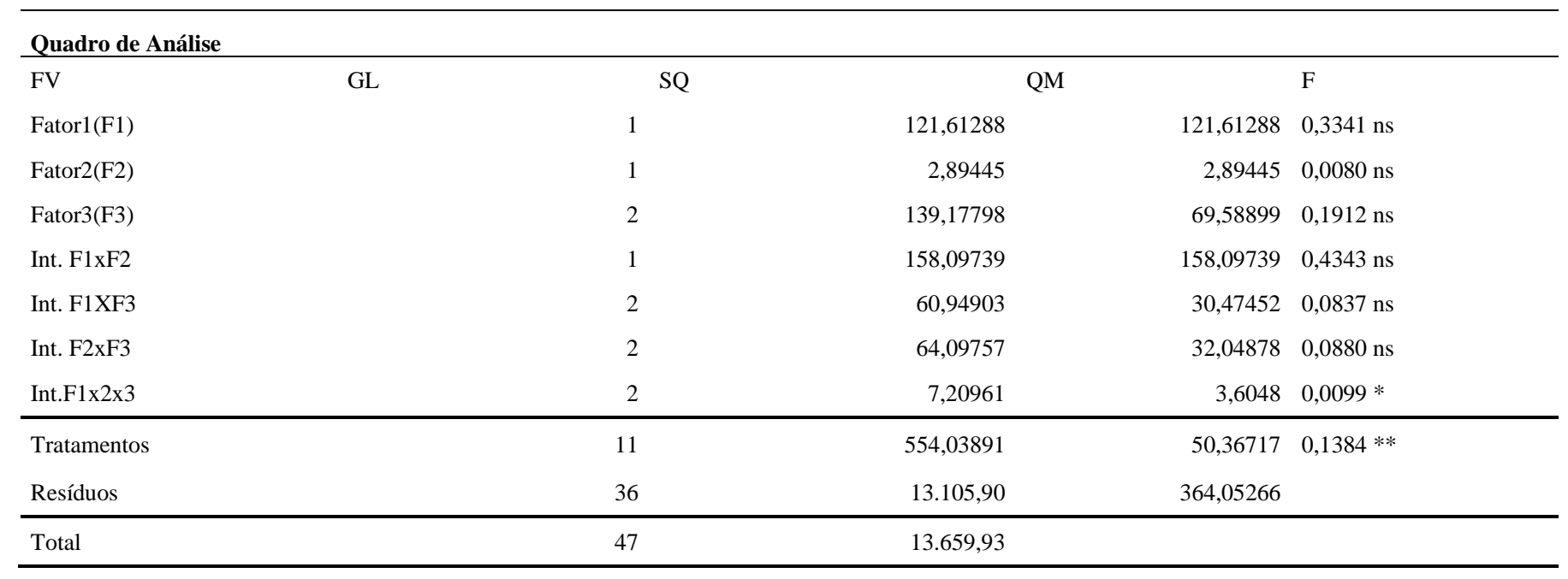

** significativo ao nível de $1 \%$ de probabilidade $(\mathrm{p}<.01)$. $*$ significativo ao nível de $5 \%$ de probabilidade $(.01=<\mathrm{p}<.05)$. ns não significativo $(\mathrm{p}>=.05)$. Fonte: Autores.

Segundo Grieser (2012) trabalhando com o crescimento e desenvolvimento de codornas de corte de 1 a 42 dias, por períodos semanais, com temperatura de 30 a $32{ }^{\circ} \mathrm{C}$ encontrou para ganho de peso o valor de 22,59 g/ave. Portanto, as codornas do segundo tratamento (T2), ocorreram perdas do incremento calórico para o ambiente, por meio dos processos sensíveis, sem gasto de energia, bem como, diminuiu o incremento no ganho de peso na temperatura mais elevada. Segue Tabela 2 com as médias de ganho de peso e conversão alimentar. 
Tabela 2. Quadro de análise de médias do Rendimento: Ganho de Peso (GP) e Conversão Alimentar (CA) em função das temperaturas estudadas.

\begin{tabular}{lcc}
\hline Análise do Rendimento & & CA (g/animal/semana) \\
\hline Temperatura & GP (g/animal/semana) & 1,05 \\
$\mathrm{~T} 1\left(25,5^{\circ} \mathrm{C}\right)$ & 40,87 & 0,87 \\
$\mathrm{~T} 2\left(30,8^{\circ} \mathrm{C}\right)$ & 37,68 & 83,63 \\
\hline $\mathbf{C V}(\%)$ & 48,58 & \\
\hline
\end{tabular}

Médias seguidas de mesma letra na coluna não diferem entre si, pelo Teste de Tukey $(\mathrm{p}>0,05)$. Fonte: Autores.

As codornas europeias apresentam ganho de peso mais rápido que as japonesas em todas as idades, o pico máximo da taxa de crescimento aos 27 dias, provável deposição de proteína e água na carcaça, depois essa taxa de crescimento diminui e o ganho de peso passa a ter um retorno decrescente, com aumento da deposição de gordura em vísceras, retenção de nutrientes no ovário - oviduto, com exigência de energia dietética, Silva et al (2011).

Observou-se que nas temperaturas, densidades e alturas não houve interação significativa quanto ao ganho de peso, mas na interação tripla (temperatura x densidade $\mathrm{x}$ altura) constatou-se efeito significativo ao nível de $5 \%$ de probabilidade com margem de segurança entre elas.

\section{Conclusão}

O ganho de peso do animal diminuiu significativamente quando aumentou a temperatura, ocorrendo diferença média de 20 g. Quando comparadas as densidades, observou-se uma diferença significativa do peso vivo do animal na primeira fase experimental.

O desempenho e características de carcaça de codornas de corte, de 16 a 42 dias de idade, são prejudicados em ambientes de $30,8^{\circ} \mathrm{C}$ e ITGU igual ou superior a 78 .

Sugerimos que trabalhos futuros continuem com a alteração da densidade de aves, mantendo as temperaturas estudadas, tentando viabilizar uma nova lotação para produção de codornas em ambiente controlado.

\section{Referências}

Abreu, V. M. A., \& Abreu, P. G. (2003). Diagnóstico bioclimático para produção de aves na mesorregião centro sul baiano. Concórdia: EMBRAPA suínos e aves. 11p. Anais... Maceió - Al, 2011.

Barreto, S. L. T., Pereira, C. A., Umigi, R. T. et al.(2007). Determinação da exigência nutricional de cálcio de codornas japonesas na fase inicial do ciclo de produção. Revista Brasileira de Zootecnia, 36(10), 68-78.

Bertechini, A. G. (2010) Situação Atual e Perspectivas Para a Coturnicultura no Brasil. In: IV Simpósio Internacional e III Congresso Brasileiro de Coturnicultura. Lavras: Anais... Lavras - MG, 2010.

Corrêa, G. S. S., Silva, M. A., Fontes, D. O., et al. (2005). Efeito de diferentes níveis deproteína e energia sobre o rendimento de carcaça de codornas européias. Arquivos Brasileiros de Medicina Veterinária e Zootecnia, 57(20), 266-271.

Dias, R. G. (2012). Efeito do sexo sobre o rendimento de carcaça de codornas de corte da linhagem EV1. Universidade Federal Minas Gerais, Belo Horizonte.

Gomes, D. L. S. (2010). Efeito do balanço eletrolítico em dietas com redução protéica sobre parâmetros fisiológicos de poedeiras semi pesadas após pico de postura. In: 47 a Reunião Anual da Sociedade Brasileira de Zootecnia, Salvador, BA.

Grieser, D. O. (2012). Estudo do crescimento e composição corporal de linhagens de codornas de corte e postura. (Mestrado em Zootecnia), Universidade Estadual de Maringá, Maringá.

Guimarães, M. C. C. (2012). Desempenho produtivo e qualidade de ovos de Codornas nas estaçães chuvosa e seca no Semiárido paraibano. (Tese de Doutorado). Universidade Federal de Campina Grande. Campina Grande, PB.

IBGE (2009). Produção da Pecuária Municipal, 37. http://www.ibge.gov.br/home/estatistica/economia/ppm/2010/ppm2010.pdf. Acesso em 21 de junho de 2014. 
Research, Society and Development, v. 10, n. 11, e540101119753, 2021

(CC BY 4.0) | ISSN 2525-3409 | DOI: http://dx.doi.org/10.33448/rsd-v10i11.19753

Jordão Filho, J. (2011). XXI Congresso Brasileiro de Zootecnia, Universidade Federal de Alagoas. Exigências nutricionais de codornas. Zootec.

Lanna, L. L. (2012). Morfologia testicular e reserva espermática na codorna japonesa (Coturnix coturnix japônica). (Tese de Doutorado). Escola de Medicina Veterinária. Universidade Federal de Minas Gerais, Belo Horizonte.

Melo, J. K. A. et al. (2013). Potencialidade e extensão da coturnicultura no município de São Bento do Una, Pernambuco. In: XII Jornada de Ensino, Pesquisa e Extensão da UFRPE. Recife. Anais... Recife.

Monte, G. L. S. et al. (2018). Parasitic profiling of Japanese quails (Coturnix japonica) on two farms with conventional production system in the Amazon region. Pesq. Vet. Bras. 38(5): 847-851.

Murakami, A. E., \& Garcia, E R. M. (2006). Novas tecnologias no sistema de produção de codornas. In: II Congresso Latino-Americano de Nutrição Animal. Anais... Palestra Técnica.

Oliveira, W. P. (2011). Redução do nível de proteína bruta em rações para frangos de corte em ambiente de termoneutralidade. Revista Brasileira de Zootecnia, 40(80), 1725-1731.

Rodrigues, P. B. (2006). Redução dos níveis de proteína e suplementação aminoacídica em rações para codornas européias (Coturnix coturnix coturnix). Revista Brasileira de Zootecnia, 35(30), 822-829.

Silva, I. J. O., \& Miranda, K. O. S. (2009). Impactos do bem-estar na produção de ovos. Thesis, n.11, p.89-115.

Silva, J. H. V., Filho, J. J., Costa, F. G. P. et al. (2011). Exigências nutricionais de codornas. In: XXI CONGRESSO BRASILEIRO DE ZOOTECNIA. Maceió: Anais... Maceió - Al.

Souza, M. S. (2013). Determinação das faixas de conforto térmico para codornas de corte de diferentes idades. ix, 76f. Universidade Federal de Viçosa.

União Brasileira De Avicultura. (2001). Relatório Anual. 51p. 